\title{
NEPENTHES KHASIANA'S CHITIN-INDUCED PITCHER LIQUID: A POTENTIAL TREATMENT FOR OPPORTUNISTIC FUNGAL INFECTION
}

JESSICA BROWN • M enlo Park • California 94025 • USA • publications.jrb@ gmail.com

Keywords: Nepenthes khasiana, fungal infection, antimycotics, napthoquinone, secondary metabolite

The endangered tropical pitcher plant Nepenthes khasiana has evolved mechanisms to lure, capture, and digest prey animals (USDA 2003; Eilenberg et al. 2010). Native to areas with nutrientpoor soil, consuming insects enables Nepenthes to gain additional nourishment (M ithofer 2011). L ocated at the ends of leaf tendrils, their pitchers' bright colors and nectar secretions are a powerful attractant for bugs (M ithofer 2011). Venturing insects slip on the pitcher's waxy mouth and fall inside, ultimately drowning in the liquid (Rischer et al. 2002). Glands located at the base of the pitcher secrete hydrolyzing enzymes (Eilenberg et al. 2006). This serves to break the arthropod's body apart so it can be absorbed by the plant as sustenance (Eilenberg et al. 2006). U nder special circumstances, Nepenthes pitcher liquid will also contain potent antifungal compounds called naphthoquinones (Eilenberg et al. 2010). Their purpose, it is believed, is to prevent captured prey from being consumed by fungi, a competing organism (Eilenberg et al. 2010). For hundreds of years, naphthoquinones have been utilized in A sia and South A merica for medicinal purposes (Babula et al. 2009). In the U nited States, emerging studies are investigating how these compounds can be produced, as well as their efficacy against opportunistic human fungal pathogens (Eilenberg et al. 2010). The importance of this research is underscored by the limited variety of antifungal drugs that are commercially available, as well as their susceptibility to pathogenic resistance and crosstolerance (K ayser et al. 2003).

Naphthoquinones are secondary metabolites produced by Nepenthes' pitcher liquid (Rischer et al. 2002). Secondary metabolites are not essential for a plant's regular growth, but aid in lesser functions like defense against herbivory (Bio-M edicine 2012). For centuries, many cultures have been utilizing this type of organic compound for medicinal applications (K aruppusamy 2009). A ntibiotics, widely used to treat bacterial infections, are secondary metabolites of mold (ePlantscience.com 2009). One major drawback of antibiotic medication is that its use can suppress the immune system by killing microbes which ward off infection (M erck 2008). Women taking antibiotics are at risk for developing candidiasis, which is named after its causal fungal strain Candida albicans (NIH 2010). A Iso known as vaginal yeast infection, candidiasis is responsible for more than 10 million doctor visits in the United States each year (M erck 2008). People with compromised immune systems, like the elderly and those with HIV /AIDs, are at risk for developing a more serious form of candidiasis (M erck 2008). This potentially fatal infection occurs when C. albican spores infiltrate the bloodstream (M erck 2008). There is a limited variety of commercial antimycotics (antifungal drugs) that are currently available (Eilenberg et al. 2010). O ver-usage of these antimycotics, which tend to operate by the same mechanism of action, is creating drug-resistant fungal strains (K ayser et al. 2003). M oreover, concomitant use of antifungal drugs is causing cross-resistance in pathogens (Eilenberg et al. 2006). Developing antimycotics which function differently than current ones is needed to ensure our continuing ability to treat fungal infections.

Eilenberg etal. (2006) demonstrated a method of inducing the production of antifungal secondary metabolites known as naphthoquinones in Nepenthes khasiana. Published by the Journal of Experimental Botany, the article entitled "Isolation and characterization of chitinase genes from 
pitchers of the carnivorous plant Nepenthes khasiana" showed that naphthoquinone production can be induced via colloidal chitin injections into closed N. khasiana pitchers. Chitin is the main component of insect exoskeleton, and is also an integral part of fungal cell walls (Collinge et al. 1993). Chitinase, an enzyme which degrades chitin, was found to be an implicit component of $\mathrm{N}$. khasiana pitcher liquid. Chitinase appears to function in both carnivory, by breaking-down insects, and defense, by degrading fungi. The liquid of closed N. khasiana pitchers (referred to as "preychallenged") did not contain naphthoquinones, but two types of chitinases were present. When immature Nepenthes pitchers were injected with chitin, napthiquinones and a third type of chitinase were detected. Chitinase was al so found in open pitchers containing prey, but naphthoquinones were not. Though the pitcher liquid does not appear to contain the potent antifungal naphthoquinones under normal circumstances, it may still exhibit antifungal activities due to the endogenous presence of chitinase. This might explain why there is "anthropological evidence of [people] using the liquid from unopened traps as a curing means" (Eilenberg et al. 2010).

In 2010, the Journal of Experimental Botany published a compelling article entitled "Induced production of antifungal naphthoquinones in the pitchers of the carnivorous plant Nepenthes khasiana". In this study, Eilenberg et al. (2010) examined the antifungal effects of naphthoquinones on various strains of human pathogenic fungi. Similar to previous experiments, the team induced naphthoquinone production by injecting closed Nepenthes khasiana pitchers with colloidal chitin. The N. khasiana pitcher liquid of chitin-induced, prey-challenged, and open pitchers containing prey was then screened for the presence of naphthoquinones. Only the chitin-induced pitcher liquid contained these secondary metabolites. The absence of naphthoquinones from open pitchers containing prey implies that chitin alone is not responsible for its production. However, there is evidence that the components of chitin are needed to form the naphthoquinone compound (Rischer et al. 2002). Eilenberg et al. postulate that injecting the pitchers with a syringe simulates a predatory presence. As previously discussed, the major natural role of secondary metabolites is to aid in defense (Biomedicine 2012). It is possible that naphthoquinones are synthesized "to avoid the fast consumption of organic compounds by competitors before being absorbed by the pitcher cells" (Eilenberg et al. 2010). That is, Nepenthes may produce naphthoquinones as a way of preventing insects from decomposing due to fungal activity before the plant is able to digest them.

The Eilenberg et al. study in 2010 demonstrated that chitin-induced pitcher liquid exerts antifungal and fungicidal effects on the yeast strain Candida albicans. The article cited an in vitro experiment in which naphthoquinone derivatives inhibited $\mathrm{C}$. albicans as effectively as Diflucan, the leading clinical yeast infection treatment (Tandon et al. 2009). Plumbagin is a naphthoquinone derivative that has been isolated and studied extensively due to its proven ability to inhibit and kill human pathogenic fungi (Eilenberg et al. 2010). The major problem with plumbagin is its high cytotoxicy and low therapeutic selectivity (Eilenberg et al. 2010). D roserone, an oxygenated derivative of plumbagin, exerted an antifungal effect on opportunistic fungal pathogens with a much lower level of toxicity. In addition, droserone demonstrated a different mechanism of action from that of mainstream antimycotics, like Diflucan. The majority of mainstream antimycotics prevent fungal growth by inhibiting fungal enzymes (K ayser et al. 2003). D roserone instead catabolizes, or breaks-down, fungal spores. This is advantageous considering the growing prevalence of antimycotic resistance ( $K$ ayser et al. 2003). In addition to inhibiting $C$. al bicans, naphthoquinones demonstrated lethality to Aspergillus, a common household fungus which causes the life-threatening disease aspergillosis (M erck 2008).

Extracting secondary metabolites from plant cell cultures can often be a difficult and inefficient process (Eilenberg et al. 2010). When injected with chitin, Nepenthes pitchers produce moderate 
amounts of medicinal compounds which require no additional purification (Eilenberg et al. 2010). In addition to serving as an antifungal agent, naphthoquinones derived from Nepenthes pitchers have the potential for a wide array of applications. These compounds are so versatile, they demonstrate antifungal, anti-cancer (Eilenberg \& al. 2010; Sandur \& al. 2006), anti-inflammatory (Lien et al. 1996), antimalarial (B iot etal. 2004), antiviral (Sacau et al. 2003), antiallergic (Lien etal. 1996), and antibacterial (Tandon etal. 2009) activities. Napthoquinones may also serve as a totally "green" insect control because of their ability to inhibit insect ecdysis, or moulting, which is essential for insect survival (Eilenberg et al. 2010). Considering all of the potential applications in which Nepenthes' chitin-induced pitcher liquid can be used, it is lucky that this tropical plant is able to produce exceptional yields of pure compounds, relatively simply.

\section{References}

B abula, P., A dam,V., Havel, L., and K izek, R. 2009. Noteworthy secondary metabolites naphthoquinones - their occurrence, pharmacological properties and analysis. Curr. Pharm. A nal. 5(1): 47-68.

Bio-M edicine. 2012. Secondary metabolites. http://www.bio-medicine.org/biology-definition/ Secondary_metabolite/, accessed $8 \mathrm{M}$ arch 2013.

Biot, C., B auer, H., Schirmer, R.H., and Davioud-Charvet, E. 2004. 5-substituted tetrazoles as bioisosteres of carboxylic acids. Bioisosterism and mechanistic studies on glutathione reductase inhibitors as antimalarials. J. M ed. Chem. 47(24): 5972-5983.

Collinge, D.B., K ragh, K.M., M ikkelsen, J.D., Nielsen, K.K., Rasmussen, U., and Vad, K. 1993. Plant chitinases. Plant J. 3(1): 31-40.

Eilenberg, H., Pnini-Cohen, S., Rahamim, Y., Sionov, E., Segal, E., Carmeli, S., and Zilberstein, A. 2010. Induced production of antifungal naphthoquinones in the pitchers of the carnivorous plant Nepenthes khasiana. J. Exp. B ot. 61(3): 911-922.

Eilenberg, H., Pnini-Cohen, S., Shuster, S., M ovtchan, A ., and Zilberstein, A . 2006. I solation and characterization of chitinase genes from pitchers of the carnivorous plant Nepenthes khasiana. J. Exp. B ot. 57(11): 2775-2784.

ePlantscience.com. 2009. An Online Botanical Encyclopedia. http://www.eplantscience.com/ index_files/biotechnology/M icrobial\%20biotechnology/Secondary\%20M etabolites/biotech_ antibiotics.php, accessed $8 \mathrm{M}$ arch 2013.

Karuppusamy, S. 2009. A review on trends in production of secondary metabolites from higher plants by in vitro tissue, organ and cell cultures. J. M ed. PI. Res. 3(13): 1222-1239.

Kayser, O., Kiderlen, A., and Croft, S. 2003. N atural products as antiparasitic drugs. Parasitol. Res. 90 Suppl 2: S55-62.

Lien, J.C., Huang, L.J., Wang, J.P., Teng, C.M., L ee, K.H., and Kuo, S.C. 1996. Synthesis and antiplatelet, antiinflammatory and antiallergic activities of 2,3-disubstituted 1,4-naphthoquinones. Chem. Pharm. Bull. (Tokyo) 44(6): 1181-1187.

M erck. 2008. The Manual Home Health Handbook. http://www.merckmanuals.com/home/skin_ disorders/fungal_skin_infections/candidiasis.html

http://www.merckmanuals.com/home/infections/fungal_infections/aspergillosis.html, accessed $8 \mathrm{M}$ arch 2013.

M ithofer, A . 2011. Carnivorous pitcher plants: insights in an old topic. Phytochem. 72(13): 16781682.

NIH. 2010. M edline Plus. http://www.niaid.nih.gov/topics/vaginalyeast/Pages/D efault.aspx, accessed $8 \mathrm{M}$ arch 2013. 
Sacau, E.P., Esteves-B raun, A., Ravelo, A.G., Ferro, E.A., Toduka, H., M ukainaka, T., and N ishino, H. 2003. Inhibitory effects of lapachol derivatives on epstein-barr virus activation. Bioorg. M ed. Chem. 11(4): 483-488.

Rischer, H., Hamm, A ., and B rigmann, G. 2002. Nepenthes insignis uses a $C_{2}$-portion of the carbon skeleton of $\mathrm{L}$-alanine acquired via its carnivorous organs, to build up the allelochemical plumbagin. Phytochem. 59(6): 603-609.

Sandur, S.K., Ichikawa, H., Sethi, G., A hn, K.S., and A ggarwal, B.B. 2006. Plumbagin (5-hydroxy2-methyl-1,4-naphthoquinone) suppresses NF-kappaB activation and NF-kappaB-regulated gene products through modulation of p65 and I kappaB al pha kinase activation, leading to potentiation of apoptosis induced by cytokine and chemotherapeutic agents. J. Biol. Chem. 281(25): 17023-17033.

Tandon, V.K ., M aurya, H.K ., Tripathi, A ., ShivaK eshava, G.B., Shukla, P.K., Srivastava, P., and Panda, D. 2009. 2,3-Disubstituted-1,4-naphthoquinones, 12H-benzo[b]phenothiazine-6,11-diones and related compounds: synthesis and biological evaluation as potential antiproliferative and antifungal agents. Eur. J. M ed. Chem. 44(3): 1086-1092.

USDA . 2003. Germplasm R esources Information N etwork. http://www.ars-grin.gov/cgi-bin/npgs/ html/taxon.pl?25155, accessed $8 \mathrm{M}$ arch 2013.

- Dionaea, Sarracenia, Nepenthes, Drosera,
Pinguicula, books, supplies, and much more
- We stock multiple sizes of most plants
- Rarre nursery-grown, imported
lowland/intermediate Nepenthes
- Most plants shipped potted and ready to grow!
www.petflytrap.com
orders@ 9 petflytrap.com
281-433-3286

